\title{
Quantitative Fractal Evaluation of Herbicide Effects on the Water-Absorbing Capacity of Superabsorbent Polymers
}

\author{
Renkuan Liao, Shumei Ren, and Peiling Yang \\ College of Water Conservancy \& Civil Engineering, China Agricultural University, Beijing, China \\ Correspondence should be addressed to Peiling Yang; yangpeiling@126.com
}

Received 15 January 2014; Revised 23 March 2014; Accepted 24 March 2014; Published 22 April 2014

Academic Editor: Haiqiang Wang

Copyright ( 2014 Renkuan Liao et al. This is an open access article distributed under the Creative Commons Attribution License, which permits unrestricted use, distribution, and reproduction in any medium, provided the original work is properly cited.

\begin{abstract}
The water absorption capacity of superabsorbent polymers (SAPs) is important for agricultural drought resistance. However, herbicides may leach into the soil and affect water absorption by damaging the SAP three-dimensional membrane structures. We used 100-mesh sieves, electron microscopy, and fractal theory to study swelling and water absorption in SAPs in the presence of three common herbicides (atrazine, alachlor, and tribenuron-methyl) at concentrations of $0.5,1.0$, and $2.0 \mathrm{mg} / \mathrm{L}$. In the sieve experiments it was found that $2.0 \mathrm{mg} / \mathrm{L}$ atrazine reduces the capacity by $9.64-23.3 \%$ at different swelling points; no significant diminution was observed for the other herbicides or for lower atrazine concentrations. We found that the hydrogel membrane pore distributions have fractal characteristics in both deionized water and atrazine solution. The $2.0 \mathrm{mg} / \mathrm{L}$ atrazine destroyed the water-retaining polymer membrane pores and reduced the water-absorbing mass by modifying its three-dimensional membrane structure. A linear correlation was observed between the fractal analysis and the water-absorbing mass. Multifractal analysis characterized the membrane pore distribution by using the range of singularity indexes $\Delta \alpha$ (relative distinguishing range of 16.54-23.44\%), which is superior to single-fractal analysis that uses the fractal dimension $D$ (relative distinguishing range of 2.5-4.0\%).
\end{abstract}

\section{Introduction}

Superabsorbent polymer (SAP) hydrogels are three-dimensional, crosslinked, linear, or branched polymers with a considerable number of hydrophilic groups that can absorb water, saline solutions, or physiological fluids in amounts as high as 10-1000 times their own weight $[1,2]$. SAPs are ideal for use in water absorption applications in agriculture, forestry, construction, sanitation, biology, and medicine [3-6]. Their rapid and reversible water absorbency and biocompatibility enable usage in arid and semiarid environments for holding rain and irrigation water for plants in sandy or loamy soils that perform poorly in drought conditions $[7,8]$. They also improve plant growth [9]. Agricultural polymers are usually applied at the root zone, and the retained water is not released from the network structure under normal physical pressure. However, when the soil is dry, the water is slowly released by root pressure, ensuring normal growth.

Large quantities of herbicides are introduced into farmland ecosystems every year; the global herbicide market accounted for half of the total pesticide market in 2010 [10].
Herbicides that migrate deep into the soil because of irrigation water and rainfall [11] may affect the water-absorbing capacity of SAPs by damaging their hydrogel membrane structure and micromorphology. Previous studies indicate that nutrient ions may reduce the absorbency of SAPs, and highly charged ions may modify SAP structures to the point of losing water-retaining capacity $[12,13]$. Despite the concerns, there have been few reports concerning herbicide effects on the water-absorbing capacity, and the mechanisms are not clear.

After water absorption and swelling of the SAP, the threedimensional hydrogel network is wrapped in a membrane structure to hold the water. The water is retained because of osmotic pressure and molecular forces. During swelling, the three-dimensional network unfolds continuously until it reaches equilibrium and stops absorbing water [14]. When the membrane structure is destroyed, the water stored in the internal network will be released, the SAP absorbency will be reduced, and the hydrogel will have a complex distribution of membrane pores. Therefore, to reveal the water-absorbing mechanism, studies on the hydrogel membrane pore 


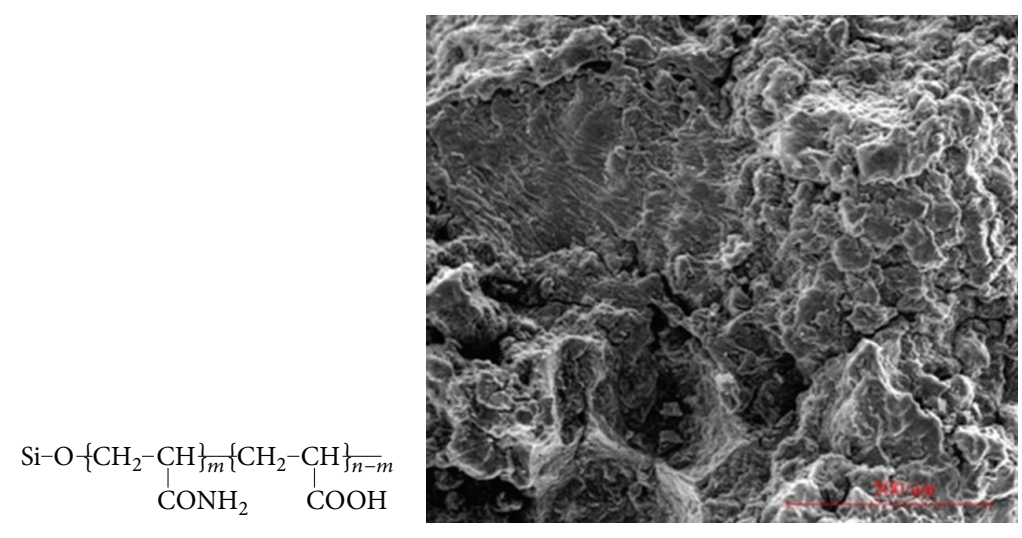

(a) Formula

(b) Surface micromorphology of dry glue

FIGURE 1: The formula and surface micromorphology of SAPs.

distributions of SAPs after absorption and swelling are very important [15]. However, the membrane pores are distributed randomly and have complicated shapes, making it difficult to correctly reveal the geometrical characteristics.

Fractal theory describes complex, natural, and nonlinear phenomena [16] and is used for shape structure characterization in non-Euclidean geometrical systems with complex structures and chaotic phenomena. Previous studies [15, 17, 18] show that, after water absorption and swelling in SAPs, the distribution of hydrogel membrane pores has fractal characteristics that can be described quantitatively. Here, fractal theory is used to characterize the micromorphology characteristics of the hydrogel and the mechanism of herbicide-induced deterioration of the SAP water-absorbing capacity. An agricultural SAP and three common herbicides are studied in 100-mesh sieve experiments, and images of the hydrogel membrane pore distribution are obtained with an environmental scanning electronic microscope (ESEM). The results provide a better understanding of the water absorption mechanisms of SAPs and enable future enhancements.

\section{Materials and Methods}

2.1. Experimental Materials. SAPs produced by HuaYe New Material Co., Ltd. (Shandong Province, China) were organicinorganic composites of attapulgite clay in polyacrylamideacrylic crosslinked polymers. They were faint yellow, 1.0$2.0 \mathrm{~mm}$ solid particles, with a moisture content $\leq 8 \%$, a deionized water uptake of $100-700 \mathrm{~g} / \mathrm{g}$, and a $0.9 \% \mathrm{NaCl}$ uptake $\geq 32 \mathrm{~g} / \mathrm{g}$. Its constitutional formula is shown in Figure 1(a) and the surface micromorphology of dry glue is shown in Figure 1(b).

Herbicides produced by American Standard Co., Ltd. (New Haven, CT, USA) were atrazine $\left(\mathrm{C}_{8} \mathrm{H}_{14} \mathrm{ClN}_{5}\right.$, $100 \%$ purity), alachlor $\left(\mathrm{C}_{14} \mathrm{H}_{20} \mathrm{ClNO}_{2}, 99.4 \%\right.$ purity), and tribenuron-methyl $\left(\mathrm{C}_{15} \mathrm{H}_{17} \mathrm{~N}_{5} \mathrm{O}_{6} \mathrm{~S}, 99.5 \%\right.$ purity).

\subsection{Experimental Methods}

2.2.1. Swelling Curves and Water Absorption Characteristics. The water absorption characteristics of SAPs were tested by 100 -mesh filtering. Solutions $(0.5,1$, and $2 \mathrm{mg} / \mathrm{L})$ of atrazine, alachlor, and tribenuron-methyl were prepared, and deionized water $(0 \mathrm{mg} / \mathrm{L})$ was used as a control. There were three replicas for each treatment. SAP $(0.30 \mathrm{~g})$ was added into $0.5 \mathrm{~L}$ solutions for swelling and filtering at time intervals of 2 , $4,6,8,10,15,20,25,30$, and 40 min (every 10 min afterward) until equilibrium was established as determined by pretests. The mass of the SAP hydrogels was determined at the elapsed times to obtain the amount of water absorption.

2.2.2. Scanning Internal Micromorphology. A FEI Quanta 200 ESEM was used to characterize the SAP structures. A cutting blade was used to obtain the middle portion of the SAP hydrogels after the water absorption tests were performed. They were imaged in low-vacuum conditions $(130 \mathrm{~Pa})$ and with an acceleration voltage of $20 \mathrm{kV}$. Each $1024 \times 678$-pixel image was stored as eight-bit grayscale files.

\subsection{Analysis Methods}

2.3.1. Fractal Dimension of Surface Topography. The fractal dimension $D$ is a characteristic parameter that quantitatively describes the complex degree [12]. Slit island analysis [16] was used to calculate the fractal dimensions of the crosssectional internal pore structure of SAP hydrogels, which can be formulated as

$$
\alpha_{D}(\varepsilon)=\frac{L^{1 / D}(\varepsilon)}{A^{1 / 2}(\varepsilon)},
$$

where $L$ denotes the pore perimeter $(\mu \mathrm{m})$; $A$ denotes the pore area $\left(\mu \mathrm{m}^{2}\right)$; and $D$ denotes the fractal dimension $\varepsilon=\eta / L_{0}$, where $\eta$ is the absolute measurement scale, $L_{0}$ is the initial perimeter of the image with constant scale of $\eta$, and $\alpha_{D}(\varepsilon)$ is a constant and is only a function of the selected scale and not the image size. The logarithm for both sides of (1) is

$$
\log L(\varepsilon)=D \log \alpha_{D}(\varepsilon)+\frac{D}{2} \log A(\varepsilon) .
$$

Image-Pro Software was used to analyze each ESEM image after digitizing and then used to measure the area and 
perimeter of each pore. $D$ is twice as large as the slope of the double-log curve between the area and the perimeter.

2.3.2. Multifractals and Singularity Spectra. Multifractal analysis takes a certain area or volume and decomposes it into a series of subdomains according to the singularity, and every subdomain forms a fractal. Multifractals not only have fractal dimensions but also have a singularity for each measurement. A multifractal singularity spectrum is the main parameter to quantitatively describe the nonuniformity, which can be expressed by $\alpha \sim f(\alpha)$ and $q \sim D(q)$. The relationship between $\alpha \sim f(\alpha)$ and $q \sim D(q)$ is obtained by the Legendre transform. The probability for the $i$ th grid can be calculated from

$$
\mu_{i}(\varepsilon)=\frac{N_{i}(\varepsilon)}{N_{t}},
$$

where $\varepsilon$ is a set of different grids with square cells that is superimposed on the whole field studied, $N_{i}(\varepsilon)$ is the measurement of $i$, and $N_{t}$ is the total measurement of the fractal set.

For multifractal distributed measurements, the partition function $\chi_{q}(\varepsilon)=\sum_{i=1}^{N(\varepsilon)} \mu_{i}(\varepsilon)^{q}$ scales with the cell size as

$$
\chi_{q}(\varepsilon) \propto \varepsilon^{\tau(q)},
$$

where $\tau(q)$ is the mass exponent of the order $q$ and can be estimated from the slope of best-fit line. The generalized fractal dimension $D(q)$ of uniformly distributed soil pores can be defined as

$$
\begin{gathered}
D(q)=\frac{\tau(q)}{(q-1)}=\lim _{\varepsilon \rightarrow 0} \frac{1}{q-1} \frac{\log \left[\sum_{i=1}^{N(\varepsilon)} \mu_{i}(\varepsilon)^{q}\right]}{\log \varepsilon} \quad(q \neq 1), \\
D_{1}=\lim _{\varepsilon \rightarrow 0} \frac{\sum_{i=1}^{N(\varepsilon)} \mu_{i}(\varepsilon) \log \mu_{i}(\varepsilon)}{\log \varepsilon} \quad(q=1) .
\end{gathered}
$$

From the Legendre transform, we obtain

$$
\begin{gathered}
\alpha(q)=\frac{\partial}{\partial q} \tau(q), \\
f(\alpha(q))=q \alpha(q)-\tau(q),
\end{gathered}
$$

where $q=0,1,2$ and $D(q)$ corresponds to the fractal capacity dimension, the information dimension, and the correlation dimension, respectively [18]. The range of $D(q)$ increases with the slope $q \sim D(q)$ and indicates that the distribution ranges of different singularity strengths of fractal structures are wider, and the nonuniformity and complexity of the measured physical quantity are increased. From the above, it can be found that $q \sim D(q)$ and $\alpha \sim f(\alpha)$ are equivalent for describing multiple fractals, and the subset of different exponents can be discerned by changing the iterative order $q$. The span of singularity indexes $\Delta \alpha=\alpha_{\text {max }}-\alpha_{\text {min }}$ of multiple fractal singularity spectra can be used to characterize the degree of nonuniformity of SAP hydrogel pores distributions [19].
The calculations have the following steps. (1) Select a grid size $\varepsilon$ and then measure and calculate the probability for each grid. (2) Choose a different $q$ and calculate the partition function $\chi_{q}(\varepsilon)$. (3) Change the grid size $\varepsilon$ and repeat the above process to calculate a series of $\chi_{q}(\varepsilon)$. (4) Trace the graph of $\ln \left(\chi_{q}(\varepsilon)\right) \sim \ln (\varepsilon)$. (5) For each $q$, calculate $\tau(q)$ by straight line fitting of $\chi_{q}(\varepsilon) \sim \varepsilon$. (6) Trace the graph of $\tau(q) \sim q$ and calculate $D_{q}$. (7) Calculate $\alpha(q)$ and $f(\alpha)$ with Legendre transforms and obtain the final multifractal spectrum $\alpha \sim$ $f(\alpha)$.

2.3.3. Data Analysis. SPSS software (15.0, one-way analysis of variance) was used for correlation and significant structure analysis.

\section{Results and Analysis}

3.1. Swelling and Absorbency of Superabsorbent Polymers. Figure 2 plots the dynamic change of hydrogel weight after water absorption by SAPs in deionized water and in three different herbicide solutions. The "parabolic" shapes of the plots indicate that the absorbency is constantly changing and that the rate gradually reduces to a swelling equilibrium point (150 min) where absorption stops. From the significance tests in Table 1, there is no appreciable reduction of the waterabsorbing mass for the SAPs $(P>0.05)$ for the three solutions of alachlor and tribenuron-methyl and for two of the atrazine solutions $(0.5,1 \mathrm{mg} / \mathrm{L})$ at the equilibrium point. Only for the $2 \mathrm{mg} / \mathrm{L}$ atrazine solution is the water-absorbing mass of the SAP significantly reduced $(P<0.05)$.

Therefore, we examined the effect of the $2 \mathrm{mg} / \mathrm{L}$ atrazine on the SAP. In Figure 2, we can see that, for the $2 \mathrm{mg} / \mathrm{L}$ atrazine solution at $10,40,70,100$, and $150 \mathrm{~min}$, the waterabsorbing mass is reduced by $17.8 \%, 23.3 \%, 15.5 \%, 10.4 \%$, and $9.64 \%$, respectively. Thus the effect of atrazine is greater during the initial phase of absorption, that is, during the first 10-40 min. The effect is reduced with continued absorption and swelling (Table 2).

3.2. Micromorphology Characteristics of Hydrogel after Water and Atrazine Absorption. Figures 3(a)-3(e) are images of the SAP hydrogel surface micromorphology after 10, 40, 70, 100, and $150 \mathrm{~min}$ of swelling in deionized water, respectively. We can see that, with the SAP water absorption and swelling, the overlapping surface is a hydrogel with water stored in its three-dimensional, crosslinked membrane structure. At longer absorption times, the membrane structure is more pronounced. When the swelling membrane pore structure increases uniformly, the SAP can hold more water. In Figures 4(a)-4(e), the same development of hydrogel micromorphology is observed in $2 \mathrm{mg} / \mathrm{L}$ atrazine. However, in the atrazine, the SAP membrane pores are not uniformly enlarged with the swelling. Large pores develop after $40 \mathrm{~min}$ and increase steadily with swelling. Compared with the hierarchical, threedimensional, crosslinked membrane structure (Figure 3(e)) in deionized water at the swelling equilibrium, there are damaging large pores in the hydrogel (Figure $4(\mathrm{e})$ ) in the $2 \mathrm{mg} / \mathrm{L}$ atrazine solution. 
TABLE 1: Water-absorbing mass (WAM) of SAP (0.3 g) at swelling equilibrium.

\begin{tabular}{lccc}
\hline Mass concentration/(mg. $\left.\mathrm{L}^{-1}\right)$ & \multicolumn{2}{c}{ Water-absorbing mass/g } & Tribenuron-methyl \\
\hline 0 & Atrazine & Alachlor & $67.27^{\mathrm{a}}$ \\
0.5 & $67.27^{\mathrm{a}}$ & $67.27^{\mathrm{a}}$ & $65.76^{\mathrm{a}}$ \\
1.0 & $63.55^{\mathrm{ab}}$ & $65.23^{\mathrm{a}}$ & $66.04^{\mathrm{a}}$ \\
2.0 & $63.49^{\mathrm{ab}}$ & $66.19^{\mathrm{a}}$ & $65.55^{\mathrm{a}}$ \\
\hline
\end{tabular}

Within the columns, values followed by the same letter are not significantly different at the 0.05 probability level.

TABLE 2: Water-absorbing mass (WAM) of SAP (0.3 g) in deionized water and atrazine solution at different swelling times.

\begin{tabular}{lccccc}
\hline Swelling time/min & & \multicolumn{2}{c}{ Atrazine mass concentration/mg $\mathrm{L}^{-1}$} & & \\
& & 0 & & WAM/g & \\
\hline 10 & WAM/g & & SD & 15.16 & \pm 0.30 \\
40 & 18.45 & \pm 0.46 & 37.00 & \pm 1.82 \\
70 & 48.21 & \pm 2.46 & 48.83 & \pm 1.45 \\
100 & 57.80 & \pm 1.41 & 55.63 & \pm 0.45 \\
150 & 62.11 & \pm 1.99 & 60.79 & \pm 1.11 \\
\hline
\end{tabular}

SD means the standard deviation.

3.3. Single Fractal Feature of Surface Micromorphology of Superabsorbent Polymers. Table 3 lists the single fractal characteristic parameters (or single fractal dimension) $D$ and the correlation coefficients $R^{2}$ from the correlative analysis of $\log L(\varepsilon) \sim \log A(\varepsilon)$ on the SAP hydrogel membrane pore distributions in deionized water and in the $2 \mathrm{mg} / \mathrm{L}$ atrazine solution at different swelling points. A significant correlation $(P<0.01)$ is observed, which indicates that it is valid to use fractal theory in the analysis of hydrogel micromorphology. In addition, we can see that, for both the deionized water and atrazine solutions, $D$ increases gradually with SAP absorption and swelling. At various swelling points, the atrazine solution reduces $D$ relative to that in deionized water. In Figure 5, there is a linear correlation $(P<0.05)$ between $D$ and the SAP water-absorbing mass, which indicates that $D$ can reflect the change in water-absorbing mass.

The water held by SAPs is mainly stored in the crosslinked membrane pore structure. The membrane structure and its pore have a direct effect on the water-absorbing capacity. Small values of the $D$ index indicate less complexity in the membrane pore distribution and less water-absorbing mass. However, we find that the use of $D$ to quantitatively characterize the micromorphology and the SAP water-absorbing capacity is limited. Comparing the different atrazine concentrations at the same state of swelling, we see that the relative discrimination is only $2.5-4.0 \%$, while for the same atrazine concentrations at different swelling times, the accumulated relative discrimination is $7.0-8.5 \%$. All of them indicate that the membrane pore distribution characteristics of the hydrogels, as analyzed by single fractal theory, are close and need a more sensitive characteristic index.

3.4. Multifractal Characteristics of Surface Micromorphology of Superabsorbent Polymer. Table 4 lists the general multifractal dimensions $D q$ and the correlation coefficients $R^{2}$ for the correlative analysis of $\ln \left(\chi_{q}(\varepsilon)\right) \sim \ln ((\varepsilon))$ for hydrogel membrane pore distributions in deionized water and the $2 \mathrm{mg} / \mathrm{L}$ atrazine solutions at different swelling points. A significant correlation $(P<0.05)$ is observed and indicates that multifractal theory is also valid in the analysis on hydrogel micromorphology. Table 5 lists the multifractal characteristic parameters (the singularity index span) $\Delta a$ for the hydrogel membrane pore distributions in deionized water and atrazine solutions at different swelling points. The linear correlation $(P<0.05)$ indicates that $\Delta a$ more clearly reflects (relative to $D$ ) the change in SAP water-absorbing mass (Figure 6). For different atrazine concentrations at the same swellings, the relative discrimination is $16.54-23.44 \%$, while for the same atrazine concentrations at different swellings, the accumulated relative discrimination is $25.02-33.04 \%$. Both are higher than those obtained from the $D$ index (above).

\section{Discussion}

The key factors for the absorbency and swelling characteristics of SAPs are the additive compounds. Liang et al. [20] examined the effect of wheat straw in SAPs and found that the highest water absorbency was obtained with $20 \%$ wheat straw in the feed. Wang et al. [21, 22] reported that the SAPs containing $15 \mathrm{wt} \%$ sodium humate had the highest water absorbency with natural guar gum, partially neutralized acrylic acid, and sodium humate as the raw materials; moreover, the absorbency was improved further by adding cation-exchanged vermiculite. Here, the SAP was produced by adding attapulgite clay to the polyacrylamideacrylic crosslinked polymer. The absorbency in deionized water is $224 \mathrm{~g} / \mathrm{g}$, which is less than that of SAPs using attapulgite clay as reported by Liu et al. [23]. That is because the SAP here is an agricultural water absorbent that requires a stable crosslinked structure to enhance reversible absorbency, 


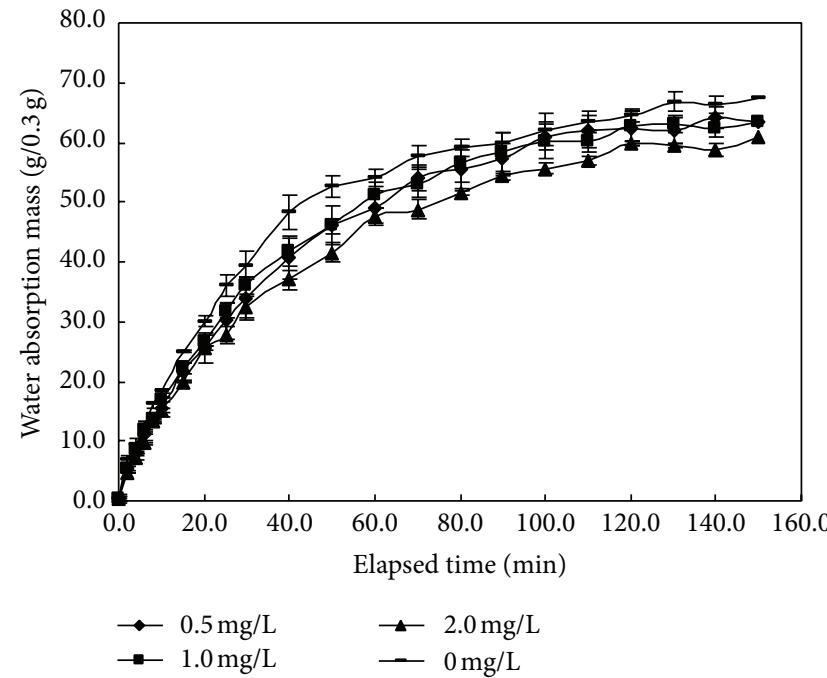

(a) Atrazine

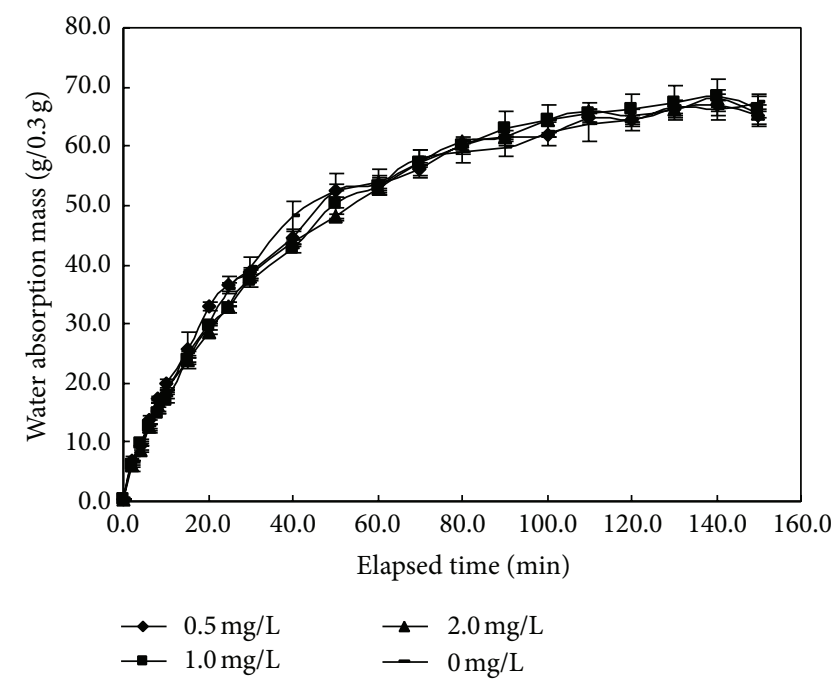

(b) Alachlor

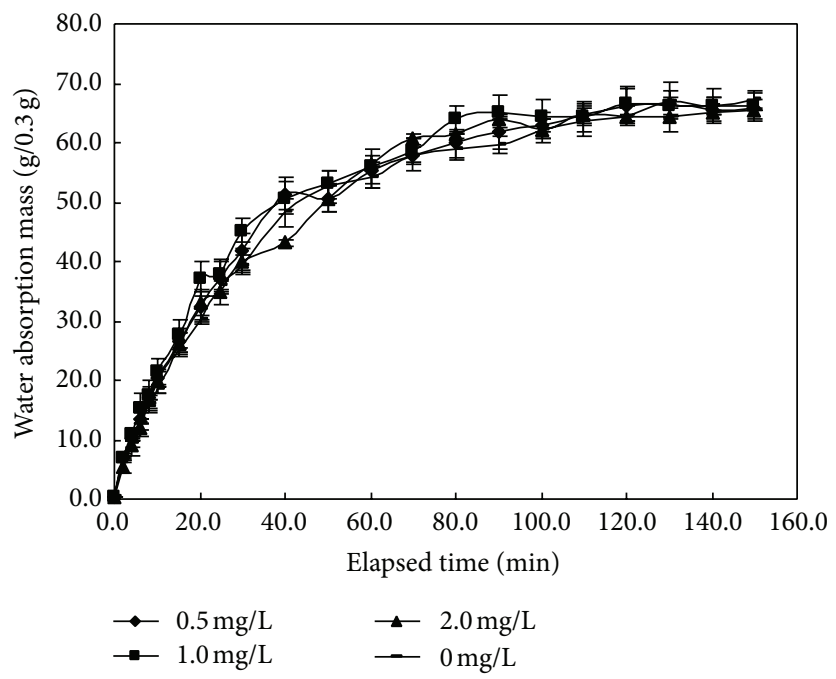

(c) Tribenuron-methyl

FIGURE 2: Swelling curves of SAP in three herbicide solutions.

TABLE 3: Single fractal dimension D of SAP hydrogel microtopography at different swelling times.

\begin{tabular}{|c|c|c|c|c|c|}
\hline \multirow{3}{*}{ Swelling time/min } & \multicolumn{4}{|c|}{ Atrazine mass concentration $/ \mathrm{mg} \cdot \mathrm{L}^{-1}$} & \multirow{3}{*}{ Relative discrimination $/ \%$} \\
\hline & \multicolumn{2}{|c|}{0} & \multicolumn{2}{|c|}{2} & \\
\hline & $D$ & $R^{2}$ & $D$ & $R^{2}$ & \\
\hline 10 & 1.072 & $0.962^{* *}$ & 1.029 & $0.951^{* *}$ & 4.0 \\
\hline 40 & 1.091 & $0.947^{* *}$ & 1.047 & $0.957^{* *}$ & 4.0 \\
\hline 70 & 1.115 & $0.952^{* *}$ & 1.081 & $0.924^{* *}$ & 3.0 \\
\hline 100 & 1.139 & $0.954^{* *}$ & 1.109 & $0.950^{* *}$ & 2.6 \\
\hline 150 & 1.151 & $0.939^{* *}$ & 1.122 & $0.956^{* *}$ & 2.5 \\
\hline Accumulated relative discrimination/\% & 7.0 & & 8.5 & & \\
\hline
\end{tabular}

Correlation is significant at the 0.01 level “**” (two-tailed).

Relative discrimination $=\left(x_{2}-x_{0}\right) / x_{0}$; accumulated relative discrimination $=\sum\left(x_{i+1}-x_{i}\right) / x_{i}$. 


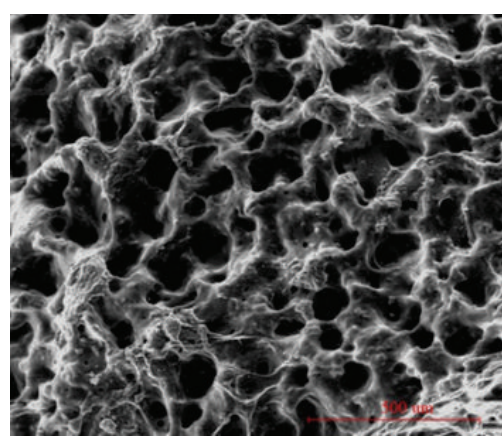

(a) $10 \mathrm{~min}$

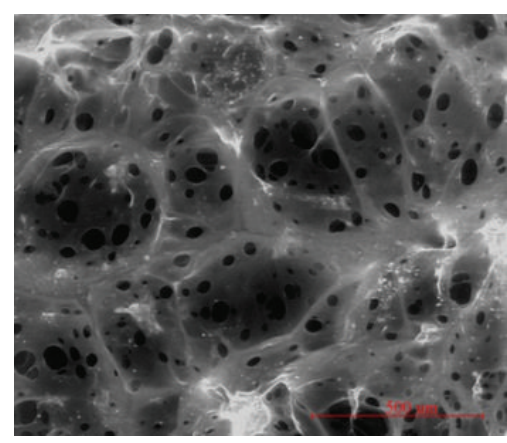

(b) $40 \mathrm{~min}$

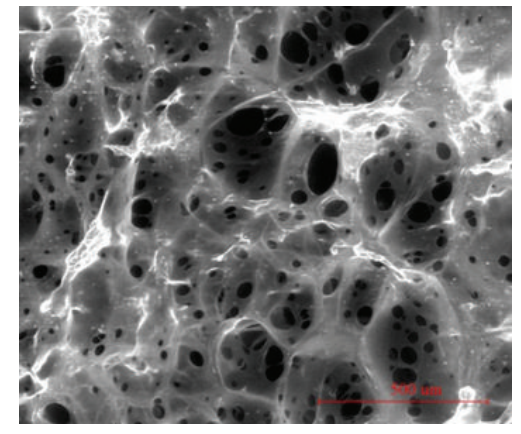

(c) $70 \mathrm{~min}$

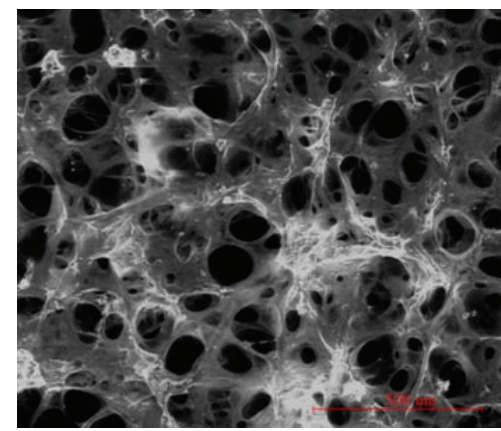

(d) $100 \mathrm{~min}$

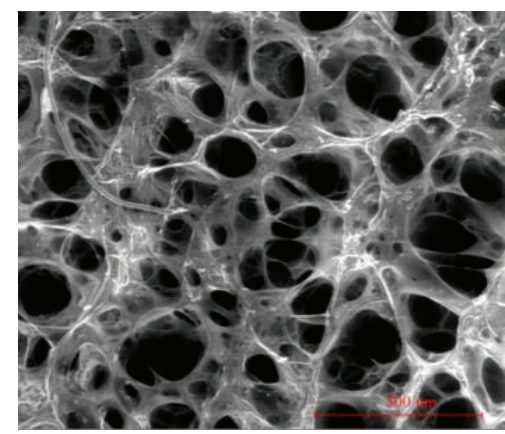

(e) $150 \mathrm{~min}$

FIGURE 3: Hydrogel microtopography of SAP in deionized water.

TABLE 4: Generalized multifractal dimension $D q$ for SAP hydrogel microtopography at different swelling times.

\begin{tabular}{|c|c|c|c|c|c|c|c|c|c|c|c|c|c|}
\hline \multirow{2}{*}{ Atrazine $/ \mathrm{mg} \cdot \mathrm{L}^{-1}$} & \multirow{2}{*}{\multicolumn{2}{|c|}{ Swelling/min }} & \multicolumn{11}{|c|}{$q$} \\
\hline & & & 1 & 3 & 5 & 7 & 9 & 0 & -1 & -3 & -5 & -7 & -9 \\
\hline \multirow{10}{*}{0} & \multirow{2}{*}{10} & $D q$ & 1.919 & 1.902 & 1.898 & 1.897 & 1.898 & 1.953 & 2.047 & 2.223 & 2.311 & 2.356 & 2.383 \\
\hline & & $R^{2}$ & $0.99^{* *}$ & $0.99^{* *}$ & $0.99^{* *}$ & $0.99^{* *}$ & $0.99^{* *}$ & $0.99^{* *}$ & $0.98^{* *}$ & $0.90^{* *}$ & $0.87^{* *}$ & $0.85^{* *}$ & $0.84^{* *}$ \\
\hline & \multirow{2}{*}{40} & $D q$ & 1.987 & 1.981 & 1.981 & 1.981 & 1.982 & 2.008 & 2.103 & 2.314 & 2.413 & 2.463 & 2.494 \\
\hline & & $R^{2}$ & $0.99^{* *}$ & $0.99^{* *}$ & $0.99^{* *}$ & $0.99^{* *}$ & $0.99^{* *}$ & $0.99^{* *}$ & $0.98^{* *}$ & $0.93^{* *}$ & $0.91^{* *}$ & $0.90^{* *}$ & $0.89^{* *}$ \\
\hline & \multirow{2}{*}{70} & $D q$ & 1.993 & 1.984 & 1.983 & 1.983 & 1.983 & 2.013 & 2.104 & 2.337 & 2.446 & 2.503 & 2.536 \\
\hline & & $R^{2}$ & $0.99^{* *}$ & $0.99^{* *}$ & $0.99^{* *}$ & $0.99^{* *}$ & $0.99^{* *}$ & $0.99^{* *}$ & $0.99^{* *}$ & $0.94^{* *}$ & $0.92^{* *}$ & $0.91^{* *}$ & $0.90^{* *}$ \\
\hline & \multirow{2}{*}{100} & $D q$ & 2.059 & 2.055 & 2.053 & 2.052 & 2.052 & 2.068 & 2.116 & 2.366 & 2.500 & 2.569 & 2.609 \\
\hline & & $R^{2}$ & $0.99^{* *}$ & $0.99^{* *}$ & $0.99^{* *}$ & $0.99^{* *}$ & $0.99^{* *}$ & $0.99^{* *}$ & $0.99^{* *}$ & $0.97^{* *}$ & $0.95^{* *}$ & $0.94^{* *}$ & $0.94^{* *}$ \\
\hline & \multirow{2}{*}{150} & $D q$ & 2.078 & 2.071 & 2.068 & 2.067 & 2.067 & 2.089 & 2.137 & 2.399 & 2.541 & 2.614 & 2.657 \\
\hline & & $R^{2}$ & $0.99^{* *}$ & $0.99^{* *}$ & $0.99^{* *}$ & $0.99^{* *}$ & $0.99^{* *}$ & $0.99^{* *}$ & $0.99^{* *}$ & $0.95^{* *}$ & $0.92^{* *}$ & $0.91^{* *}$ & $0.91^{* *}$ \\
\hline \multirow{10}{*}{2} & \multirow{2}{*}{10} & $D q$ & 1.806 & 1.800 & 1.803 & 1.805 & 1.808 & 1.838 & 1.927 & 2.063 & 2.125 & 2.157 & 2.176 \\
\hline & & $R^{2}$ & $0.99^{* *}$ & $0.99^{* *}$ & $0.99^{* *}$ & $0.99^{* *}$ & $0.99^{* *}$ & $0.99^{* *}$ & $0.95^{* *}$ & $0.83^{*}$ & $0.79^{*}$ & $0.77^{*}$ & $0.76^{*}$ \\
\hline & \multirow{2}{*}{40} & $D q$ & 1.821 & 1.814 & 1.816 & 1.819 & 1.821 & 1.853 & 1.949 & 2.102 & 2.170 & 2.203 & 2.223 \\
\hline & & $R^{2}$ & $0.99^{* *}$ & $0.99^{* *}$ & $0.99^{* *}$ & $0.99^{* *}$ & $0.99^{* *}$ & $0.99^{* *}$ & $0.96^{* *}$ & $0.86^{* *}$ & $0.83^{*}$ & $0.82^{*}$ & $0.81^{*}$ \\
\hline & \multirow{2}{*}{70} & $D q$ & 1.941 & 1.934 & 1.933 & 1.932 & 1.932 & 1.962 & 2.040 & 2.219 & 2.301 & 2.343 & 2.369 \\
\hline & & $R^{2}$ & $0.99^{* *}$ & $0.99^{* *}$ & $0.99^{* *}$ & $0.99^{* *}$ & $0.99^{* *}$ & $0.99^{* *}$ & $0.97^{* *}$ & $0.90^{* *}$ & $0.88^{* *}$ & $0.87^{* *}$ & $0.86^{* *}$ \\
\hline & \multirow{2}{*}{100} & $D q$ & 2.006 & 2.001 & 2.002 & 2.003 & 2.004 & 2.023 & 2.096 & 2.282 & 2.380 & 2.430 & 2.460 \\
\hline & & $R^{2}$ & $0.99^{* *}$ & $0.99^{* *}$ & $0.99^{* *}$ & $0.99^{* *}$ & $0.99^{* *}$ & $0.99^{* *}$ & $0.98^{* *}$ & $0.90^{* *}$ & $0.87^{* *}$ & $0.85^{* *}$ & $0.84^{* *}$ \\
\hline & \multirow{2}{*}{150} & $D q$ & 2.008 & 2.005 & 2.004 & 2.005 & 2.005 & 2.023 & 2.106 & 2.329 & 2.433 & 2.486 & 2.518 \\
\hline & & $R^{2}$ & $0.99^{* *}$ & $0.99^{* *}$ & $0.99^{* *}$ & $0.99^{* *}$ & $0.99^{* *}$ & $0.99^{* *}$ & $0.99^{* *}$ & $0.93^{* *}$ & $0.92^{* *}$ & $0.91^{* *}$ & $0.90^{* *}$ \\
\hline
\end{tabular}

Correlation is significant at the 0.01 level “**” and 0.05 level “*” (two-tailed). 
TABLE 5: Multifractal characteristic parameter $\Delta a$ of SAP hydrogel microtopography at different swelling times.

\begin{tabular}{|c|c|c|c|c|c|c|c|}
\hline \multirow{3}{*}{ Swelling time/min } & \multicolumn{6}{|c|}{ Atrazine mass concentration $/ \mathrm{mg} \cdot \mathrm{L}^{-1}$} & \multirow{3}{*}{ Relative discrimination $/ \%$} \\
\hline & \multicolumn{3}{|c|}{0} & \multicolumn{3}{|c|}{2} & \\
\hline & $a(q)_{\max }$ & $a(q)_{\min }$ & $\Delta a$ & $a(q)_{\max }$ & $a(q)_{\min }$ & $\Delta a$ & \\
\hline 10 & 2.491 & 1.898 & 0.593 & 2.254 & 1.800 & 0.454 & 23.44 \\
\hline 40 & 2.616 & 1.980 & 0.636 & 2.306 & 1.814 & 0.492 & 22.64 \\
\hline 70 & 2.671 & 1.982 & 0.689 & 2.470 & 1.932 & 0.538 & 21.92 \\
\hline 100 & 2.775 & 2.052 & 0.723 & 2.582 & 2.001 & 0.581 & 19.64 \\
\hline 150 & 2.834 & 2.066 & 0.768 & 2.646 & 2.004 & 0.642 & 16.54 \\
\hline Accumulated relative discrimination/\% & & & 25.02 & & & 33.04 & \\
\hline
\end{tabular}

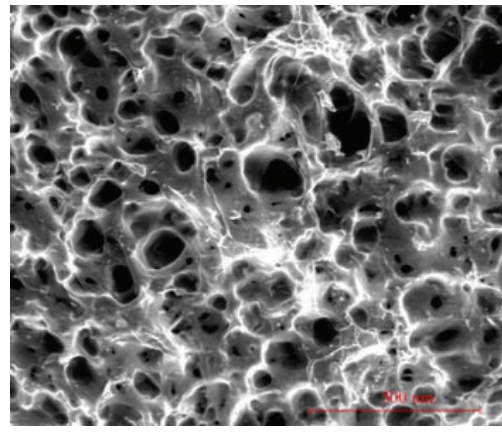

(a) $10 \mathrm{~min}$

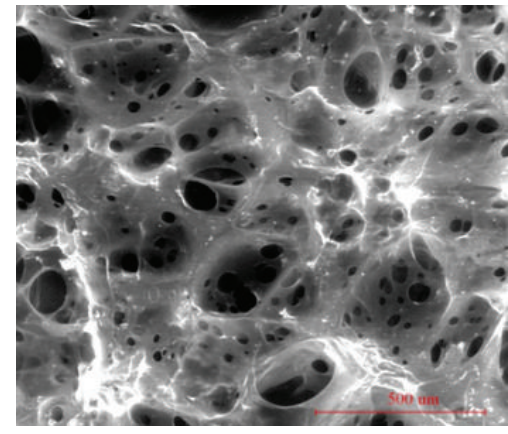

(b) $40 \mathrm{~min}$

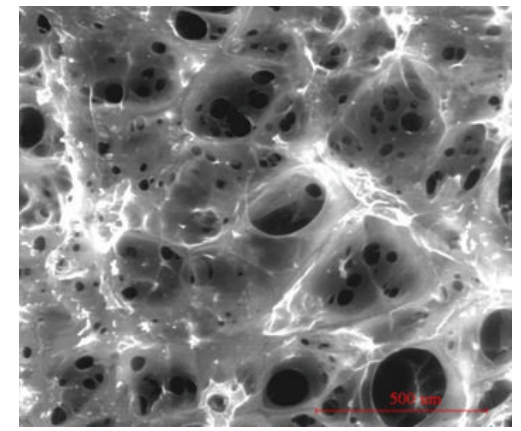

(c) $70 \mathrm{~min}$

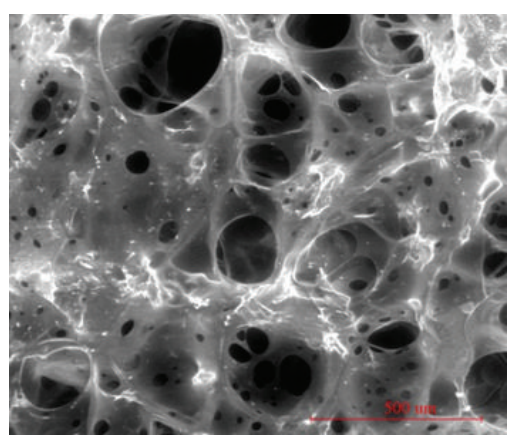

(d) $100 \mathrm{~min}$

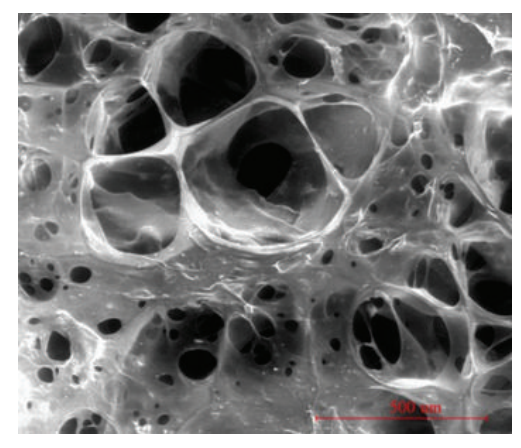

(e) $150 \mathrm{~min}$

FIgURE 4: Hydrogel microtopography of SAP in $2 \mathrm{mg} / \mathrm{L}$ atrazine solutions.

rather than attapulgite clay. The absorbency of SAPs is influenced by many other factors, such as particle size, temperature, soil texture $[24,25]$, and other soil conditions. Electrolytes in soils can have appreciable impact on SAP absorbency, depending on ion type and concentration [26, 27]. Because SAPs have hydrophilic groups, they may have a stronger interaction with polar molecules and thus make the absorbency more sensitive. Here, the $2 \mathrm{mg} / \mathrm{L}$ atrazine solution significantly reduces the water-absorbing mass of the SAP. ESEM imaging reveals that, relative to those structures in deionized water, damaged membrane structures are observed in $2 \mathrm{mg} / \mathrm{L}$ atrazine solution. These are caused by hydrogen bonding and Van der Waals interactions among the atrazine and the hydroxyl and carboxyl groups of the SAP [28]. These interactions induce large-scale damage to the membrane, resulting in reduced water-absorbing mass.
In the fractal analysis, $\Delta a$ becomes larger with SAP water absorption and swelling. At various swelling points in $2 \mathrm{mg} / \mathrm{L}$ atrazine, all $\Delta a$ indices for the hydrogel microtopography are smaller relative to deionized water. This correlates with the $D$ index observations and with the change in water-absorbing mass. In contrast to the $2.5-4.0 \%$ relative discrimination for the $D$ index, that of $\Delta a$ is $16.54 \%-23.44 \%$. Thus fractal analysis over multiple scales is more sensitive than that in a single scale. In addition, multifractal analysis can reveal specific structures and characteristics induced by different local conditions or levels. It will reveal overall features from part of the system, which is more accurate than a single fractal describing complex morphology $[19,29,30]$. Moreover, no other quantification methods are effective in evaluating the hydrogel membrane pore distribution. The fractal analysis supports the notion that the membrane pore 


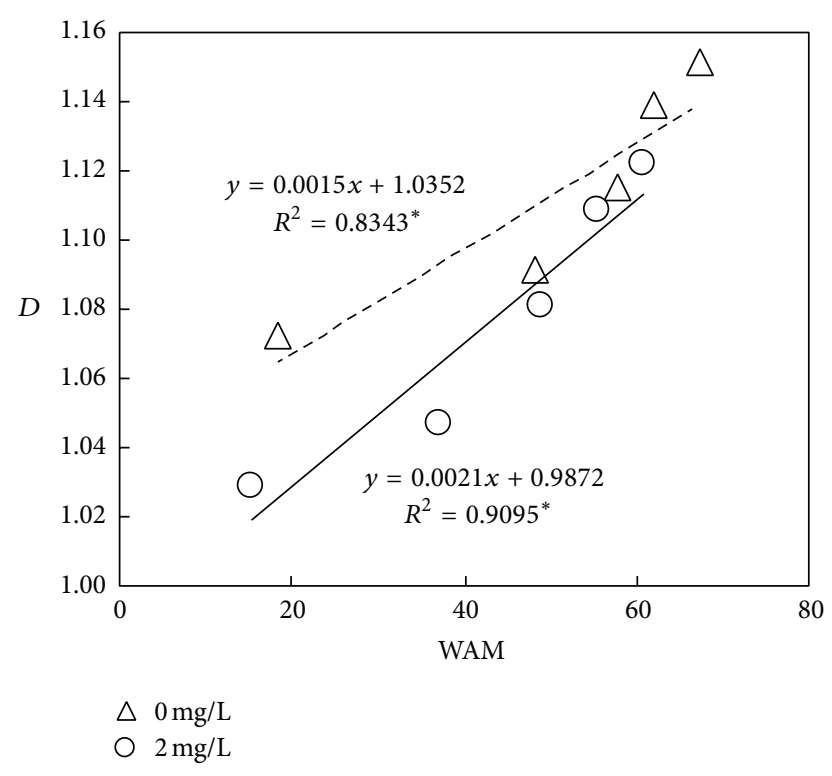

FIGURE 5: Correlation analysis between $D$ and WAM.

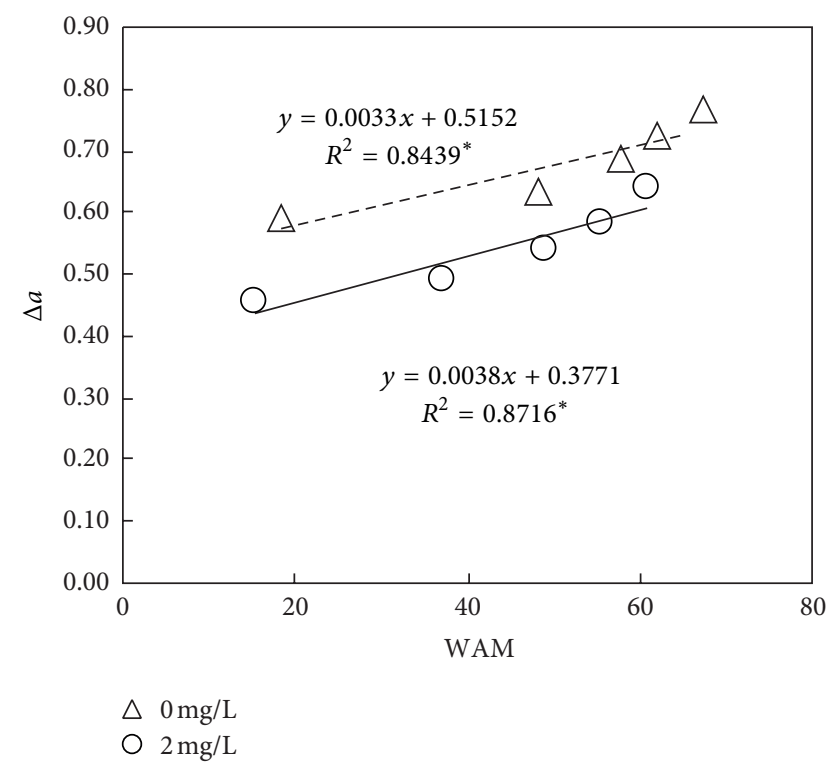

Figure 6: Correlation analysis between $\Delta a$ and WAM.

three-dimensional structure unfolds continuously during water absorption and swelling [14]. Both the $D$ and $\Delta a$ indices in the $2 \mathrm{mg} / \mathrm{L}$ atrazine solution are less than those in deionized water, which confirms the notion that the forces between the atrazine and the hydroxyls and carboxyls in the SAP reduce the complexity of the membrane structure [28]. All of these conclusions are strongly supported by the SAP water-absorbing mass data. We can conclude that the combination of image analysis and fractal theory can be used to quantitatively evaluate the water-absorbing capacity of SAPs and could be applied to other areas in environmental and materials science. Fractal theory could also be used for evaluating nonlinear processes, such as soil pore distributions for soil permeability and complex crystal structures. Finally, it could be used to design structures for materials fabrication that would have unique features.

\section{Conclusions}

(1) Alachlor and tribenuron-methyl herbicides at concentrations of $0.5,1$, and $2 \mathrm{mg} / \mathrm{L}$ have no observable effects on the water-absorbing capacity of SAPs. Similarly, 0.5 and $1.0 \mathrm{mg} / \mathrm{L}$ solutions of atrazine have no effect. Only at $2 \mathrm{mg} / \mathrm{L}$ atrazine concentration will the SAP water-absorbing mass be significantly reduced. The effect gradually weakens with swelling of the SAP, and the relative reduction is $9.64 \%-23.3 \%$.

(2) The micromorphology of the hydrogel is changed to a hierarchical, three-dimensional, crosslinked membrane structure following SAP swelling in deionized water. The membrane pores grow uniformly with water absorption and swelling. However, in the $2.0 \mathrm{mg} / \mathrm{L}$ atrazine solution, larger pores are observed during swelling tales and the membrane structure is destroyed at the swelling equilibrium point.

(3) Fractal analysis reveals clear differences in the hydrogel membrane pore distributions formed during water absorption and swelling in deionized water versus $2.0 \mathrm{mg} / \mathrm{L}$ atrazine. The single fractal dimension $D$ index gradually increases less with swelling in atrazine solution relative to that in deionized water. Along with the micromorphology characteristics of the hydrogel, the $2.0 \mathrm{mg} / \mathrm{L}$ atrazine solution may destroy the membrane structure that retains the water. This reduces both the complexity of the threedimensional membrane structure and the waterabsorbing mass of the SAP.

(4) Compared with single fractal analysis, multifractal analysis enables a more detailed characterization of the hydrogel membrane pore distribution and improves the ability to distinguish effects of the atrazine on the SAP water-absorbing mass from 2.5$4.0 \%$ to $16.54-23.44 \%$. Furthermore, both the $D$ and $\Delta a$ indices exhibit a significant linear correlation with the water-absorbing mass.

\section{Conflict of Interests}

The authors declare that there is no conflict of interests regarding the publication of this paper.

\section{Acknowledgment}

This work was financially supported by the National Natural Science Foundation of China (51379210).

\section{References}

[1] M. J. Ramazani-Harandi, M. J. Zohuriaan-Mehr, A. A. Yousefi, A. Ershad-Langroudi, and K. Kabiri, "Rheological determination of the swollen gel strength of superabsorbent polymer hydrogels," Polymer Testing, vol. 25, no. 4, pp. 470-474, 2006. 
[2] A. Pourjavadi, M. Ayyari, and M. S. Amini-Fazl, "Taguchi optimized synthesis of collagen-g-poly(acrylic acid)/kaolin composite superabsorbent hydrogel," European Polymer Journal, vol. 44, no. 4, pp. 1209-1216, 2008.

[3] F. L. Zhang, M. Z. Liu, M. Y. Guo, and L. Wu, "Preparation of superabsorbent polymer with slow-release phosphate fertilizer," Journal of Applied Polymer Science, vol. 92, no. 5, pp. 3417-3421, 2004.

[4] A. Hüttermann, L. J. B. Orikiriza, and H. Agaba, "Application of superabsorbent polymers for improving the ecological chemistry of degraded or polluted lands," Clean-Soil, Air, Water, vol. 37, no. 7, pp. 517-526, 2009.

[5] M. A. Molina, C. R. Rivarola, and C. A. Barbero, "Study on partition and release of molecules in superabsorbent thermosensitive nanocomposites," Polymer, vol. 53, no. 2, pp. 445453, 2012.

[6] A. Hebeish, M. Hashem, M. M. El-Hady et al., "Development of CMC hydrogels loaded with silver nano-particles for medical applications," Carbohydrate Polymers, vol. 92, no. 1, pp. 407-413, 2013.

[7] J. Akhter, K. Mahmood, K. A. Malik, A. Mardan, M. Ahmad, and M. M. Iqbal, "Effects of hydrogel amendment on water storage of sandy loam and loam soils and seedling growth of barley, wheat and chickpea," Plant, Soil and Environment, vol. 50, no. 10, pp. 463-469, 2004.

[8] V. Arbona, D. J. Iglesias, J. Jacas, E. Primo-Millo, M. Talon, and A. Gómez-Cadenas, "Hydrogel substrate amendment alleviates drought effects on young citrus plants," Plant and Soil, vol. 270, no. 1, pp. 73-82, 2005.

[9] S. S. Dorraji, A. Golchin, and S. Ahmadi, "The effects of hydrophilic polymer and soil salinity on corn growth in sandy and loamy soils," Clean-Soil, Air, Water, vol. 38, no. 7, pp. 584$591,2010$.

[10] L. Zhao, S. M. Luo, H. S. Li et al., "Eco-toxicological effects of four herbicides on typical aquatic snail Pomacea canaliculata and Crown conchs," Acta Ecologica Sinica, vol. 31, no. 19, pp. 5720-5727, 2011.

[11] V. C. Albright III, I. J. Murphy, J. A. Anderson et al., "Fate of atrazine in switchgrass-soil column system," Chemosphere, vol. 90, no. 6, pp. 1847-1853, 2013.

[12] B. Zhou, R. K. Liao, Y. K. Li et al., "Water-absorption characteristics of organic-inorganic composite superabsorbent polymers and its effect on summer maize root growth," Journal of Applied Polymer Science, vol. 126, pp. 423-435, 2012.

[13] W. B. Wang and A. Q. Wang, "Nanocomposite of carboxymethyl cellulose and attapulgite as a novel $\mathrm{pH}$-sensitive superabsorbent: synthesis, characterization and properties," Carbohydrate Polymers, vol. 82, no. 1, pp. 83-91, 2010.

[14] T. K. Mudiyanselage and D. C. Neckers, "Highly absorbing superabsorbent polymer," Journal of Polymer Science A: Polymer Chemistry, vol. 46, no. 4, pp. 1357-1364, 2008.

[15] Y. K. Li, T. W. Xu, Z. Y. Ouyang et al., "Micromorphology of macromolecular superabsorbent polymer and its fractal characteristics," Journal of Applied Polymer Science, vol. 113, no. 6, pp. 3510-3519, 2009.

[16] B. B. Mandelbrot, D. E. Passoja, and A. J. Paullay, "Fractal character of fracture surfaces of metals," Nature, vol. 308, no. 5961, pp. 721-722, 1984.

[17] A. Jurjiua, T. Koslowskib, C. von Ferbera et al., "Dynamics and scaling of polymer networks: vicsek fractals and hydrodynamic interactions," Chemical Physics, vol. 294, no. 2, pp. 187-199, 2003.
[18] A. N. D. Posadas, D. Giménez, R. Quiroz, and R. Protz, "Multifractal characterization of soil pore systems," Soil Science Society of America Journal, vol. 67, no. 5, pp. 1361-1369, 2003.

[19] Y. K. Li, Y. Z. Liu, G. B. Li et al., "Surface topographic characteristics of suspended particulates in reclaimed wastewater and effects on clogging in labyrinth drip irrigation emitters," Irrigation Science, vol. 30, no. 1, pp. 43-56, 2012.

[20] R. Liang, H. B. Yuan, G. X. Xi et al., "Synthesis of wheat strawg-poly(acrylic acid) superabsorbent composites and release of urea from it," Carbohydrate Polymers, vol. 77, no. 2, pp. 181-187, 2009.

[21] W. B. Wang and A. Q. Wang, "Synthesis, swelling behaviors, and slow-release characteristics of a guar gum-g-poly(sodium acrylate)/sodium humate superabsorbent," Journal of Applied Polymer Science, vol. 112, no. 4, pp. 2102-2111, 2009.

[22] W. B. Wang, N. H. Zhai, and A. Q. Wang, "Preparation and swelling characteristics of a superabsorbent nanocomposite based on natural guar gum and cation-modified vermiculite," Journal of Applied Polymer Science, vol. 119, no. 6, pp. 3675-3686, 2011.

[23] R. F. Liu, J. P. Zhang, A. Q. Wang et al., "Water absorbency and its influence factor of PAM-atta superabsorbent composite," Transactions of the Chinese Society of Agricultural Engineering, vol. 21, no. 9, pp. 47-50, 2005.

[24] K. Kabiri, S. Hesarian, M. J. Zohuriaan-Mehr et al., "Superabsorbent polymer composites: does clay always improve properties?" Journal of Materials Science, vol. 46, no. 20, pp. 6718-6725, 2011.

[25] J. Yu, I. Shainberg, Y. L. Yan, J. G. Shi, G. J. Levy, and A. I. Mamedov, "Superabsorbents and semiarid soil properties affecting water absorption," Soil Science Society of America Journal, vol. 75, no. 6, pp. 2305-2313, 2011.

[26] Y. Zhao, J. Kang, and T. W. Tan, "Salt-, pH- and temperatureresponsive semi-interpenetrating polymer network hydrogel based on poly(aspartic acid) and poly(acrylic acid)," Polymer, vol. 47, no. 22, pp. 7702-7710, 2006.

[27] H. A. Abd El-Rehim, E.-S. A. Hegazy, and H. L. Abd El-Mohdy, "Radiation synthesis of hydrogels to enhance sandy soils water retention and increase plant performance," Journal of Applied Polymer Science, vol. 93, no. 3, pp. 1360-1371, 2004.

[28] W. C. Yang, Q. Q. Wang, and W. P. Liu, "Adsorption of herbicide atrazine on soils," Chinese Journal of Environmental Science, vol. 21, no. 4, pp. 94-97, 2000.

[29] J. J. Francisco, G. RavéE, J. V. Giráldez et al., “The influence of the geometry of idealised porous media on the simulated flow velocity: a multifractal description," Geoderma, vol. 150, no. 1-2, pp. 196-204, 2009.

[30] N. Bird, M. C. Díaz, A. Saa, and A. M. Tarquis, "Fractal and multifractal analysis of pore-scale images of soil," Journal of Hydrology, vol. 322, no. 1-4, pp. 211-219, 2006. 

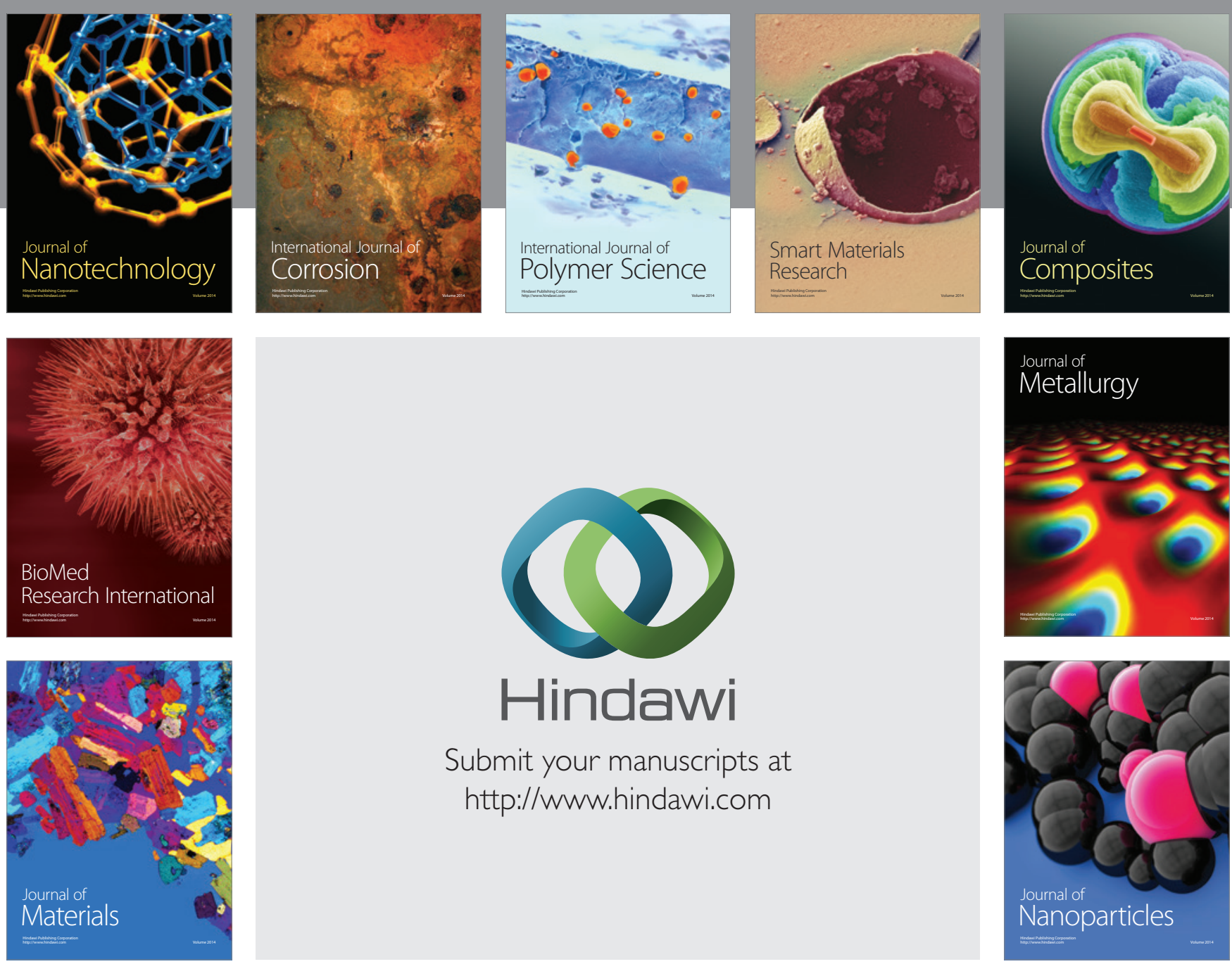

Submit your manuscripts at http://www.hindawi.com
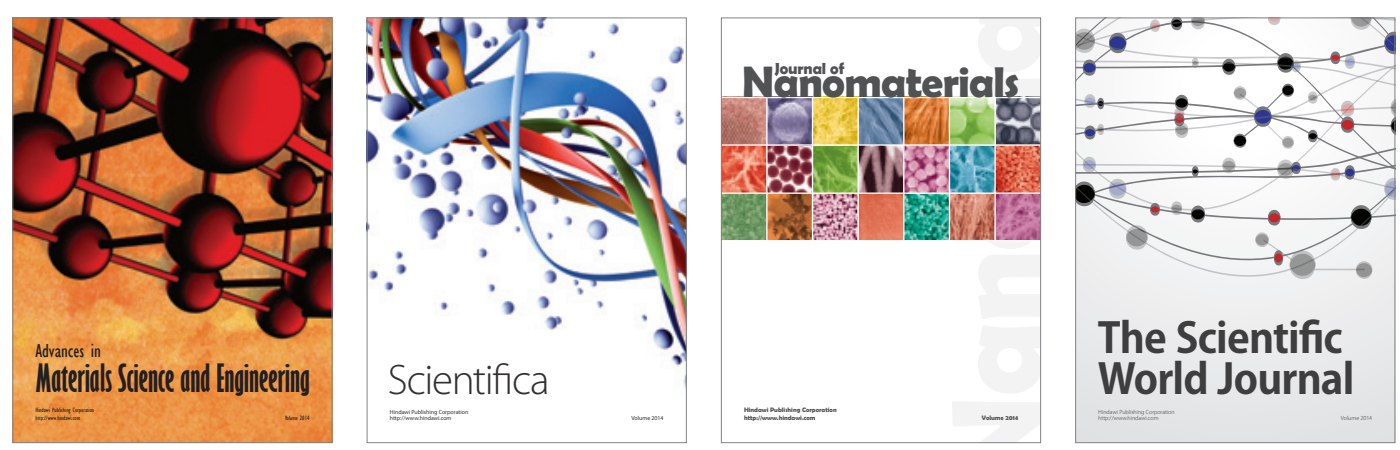

\section{The Scientific World Journal}
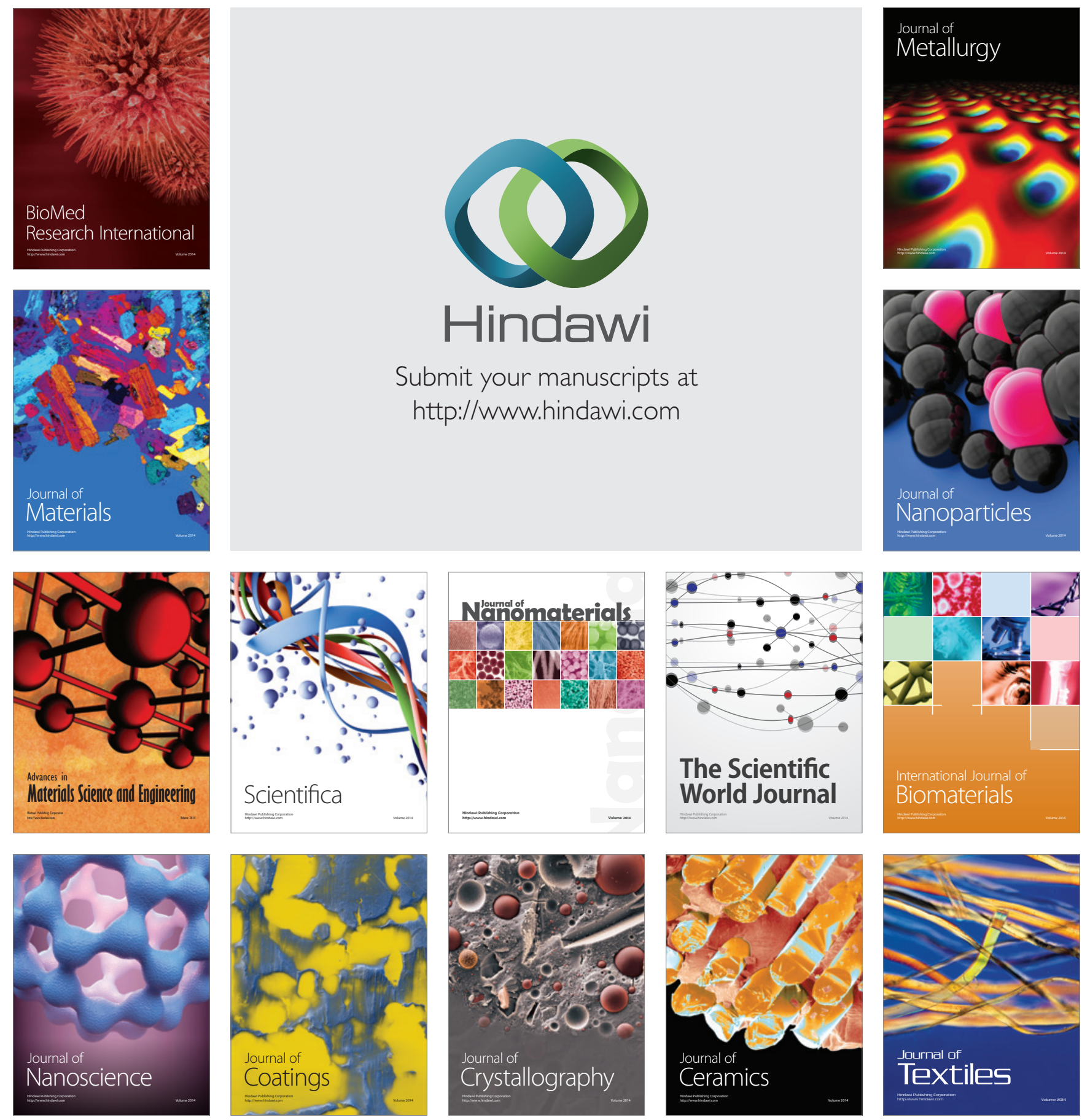